\title{
Effect of fibre content and alkali treatment on mechanical properties of Roystonea regia-reinforced epoxy partially biodegradable composites
}

\author{
GOVARDHAN GOUD* and R N RAO* \\ Department of Mechanical Engineering, National Institute of Technology, Warangal 506 004, India
}

MS received 12 June 2010; revised 12 July 2010

\begin{abstract}
The present paper investigates the effect of fibre content and alkali treatment on tensile, flexural and impact properties of unidirectional Roystonea regia natural-fibre-reinforced epoxy composites which are partially biodegradable. The reinforcement Roystonea regia (royal palm) fibre was collected from the foliage of locally available royal palm tree through the process of water retting and mechanical extraction. The poor adhesion between fibre and matrix is commonly encountered problem in natural-fibre-reinforced composites. To overcome this problem, specific physical and chemical treatments were suggested for surface modification of fibres by investigators. Alkali treatment is one of the simple and effective surface modification techniques which is widely used in natural fibre composites. In the present study both untreated and alkali-treated fibres were used as reinforcement in Roystonea regia epoxy composites and the tensile, flexural and impact properties were determined at different fibre contents. The alkali treatment found to be effective in improving the tensile and flexural properties while the impact strength decreased.
\end{abstract}

Keywords. Roystonea regia fibre; epoxy resin; alkali treatment; mechanical properties.

\section{Introduction}

Today polymer composites are used for a wide range of applications. However, they do not undergo biodegradation easily, resulting in generation of solid waste, which causes environmental pollution. To meet this challenge, researchers are focusing their attention on producing biodegradable composites with natural fibres/fabrics. Such composites are termed as green composites. By using sisal, banana, bamboo, coir, pineapple leaf fibre, etc., several green composites have already been developed and their properties have been studied. Joseph et al (1996) studied the effect of chemical treatment on the tensile properties of short sisal fibre reinforced polyethylene composites and Shin et al (1989) investigated the mechanical properties of bamboo-epoxy composites. Joseph et al (2002) developed phenol formaldehyde composites reinforced with banana fibres and pineapple leaf fibre reinforced polyethylene composites have been developed by George et al (1998). Rout et al (2001a) investigated the influence of fibre surface modification on the mechanical properties of coir-polyester composites.

Natural fibre composites are not only biodegradable and renewable but also possess several other advantages

\footnotetext{
*Author for correspondence

(pgovardhan0@yahoo.com; rnraonitw@gmail.com)
}

such as lightweight, low cost, high specific strength, high modulus, reduced tool wear and safe manufacturing process when compared with synthetic fibre composites. Several applications of natural fibre composites can be found in construction, packaging, furniture and automotive fields. Most of the interiors of the automobiles, like door panels, trunk liners, seal backs, packages, speaker trays, engine and transmission covers, are made using natural fibre composites.

Despite several merits they also possess few drawbacks such as poor wettability, incompatibility with some polymeric matrices and high moisture absorption by the fibres (Batchiar et al 2008). The greatest challenge in using the natural fibre as reinforcement in polymer matrix is the poor adhesion between natural fibre and matrix. This results in inferior strength of the composites. The main reason for the poor compatibility is that while polymer matrix is hydrophobic and non-polar, the natural fibres are hydrophilic and have polar groups in their structure. Moreover, natural fibres also consist of several elementary fibres associated with cellulose, hemicellulose, pectin, lignin, etc. Hence, they can not be considered as the mono-filament fibres. To remove the unwanted elements from the fibre, specific treatments are necessary. Many investigators found and reported that the interfacial bonding can be enhanced by the surface modification of the fibre through alkali treatment and treatment with coupling agents, which in turn will enhance the overall per- 
formance of the composites. Sinha and Rout (2009) studied the effect of alkali treatment on jute fibre composites and reported improvement in flexural properties. Rout et al (2001) reported that after 2\% alkali treatment, coir fibre-polyester composites shown improved tensile properties. There is a $15 \%$ increase in tensile strength after alkali treatment of unidirectional sisal-reinforced epoxy composites (Rong et al 2001). Huda et al (2008), in their investigation, pointed out that surface treated fibrereinforced composites shown superior mechanical properties as compared to the untreated fibre-reinforced composites. Bisanda and Ansell (1991) studied the effect of silanes on mechanical properties of sisal-epoxy composites and found considerable enhancement in compressive strength. Similarly, surface modification treatments are found to be effective in improving the properties of composites reinforced with natural fibres such as pineapple leaf fibres (Theepopnatkul et al 2009), Hildegardia fabric (Guduri et al 2006) and oil palm fibres (Sreekala and Thomas 2003). Valadez-Gonzalez et al (1999) investigated the effect of fibre surface treatment on the fibre-matrix bond strength of natural fibrereinforced composites and reported improvement in bond strength.

In the present study, Roystonea regia fibres are used as reinforcement. They are very-low-density fibres whose extraction from Roystonea regia tree is very simple as its gum present in the sheath dissolves easily. Also, the process is inexpensive. Roystonea regia trees (also known as royal palm trees) are very popular as decorative trees due to their beauty and grace. In older references the genus of the tree was referred to as Oreodoxa. They are abundantly found in Caribbean, Central and South America, Florida, Texas and California. Foliage is the source of fibre, which falls on the ground when ripens. Some of the basic properties of this fibre were investigated by Rao et al (2007). Epoxy was used as the matrix material in the present study. The role of the matrix is to keep the fibres in a desired location, orientation and to effectively transfer the stress to fibres. Also, they protect the fibres from mechanical and chemical damage. Epoxy is most commonly used matrix material because of its high strength, low viscosity, low flow rate, low volatility during cure, low shrink rate, etc. The fibre-matrix interface plays an important role on the transformation of load from the matrix to the fibre. Although mechanical properties of the natural fibres are much lower than those of synthetic fibres such as carbon, aramid, glass, etc., their specific properties, especially stiffness, are comparable. But natural fibres are very light in weight when compared with the synthetic fibres. Most of the natural fibres are nearly $50 \%$ lighter than glass.

The present work investigated the effect of fibre content in weight percentage and alkali treatment (with $\mathrm{NaOH}$ ) on tensile, flexural and impact properties of Roystonea regia natural fibre-epoxy composites.

\section{Experimental}

\subsection{Materials}

The matrix system used is an epoxy resin (Lapox-12) and hardener (k-6) supplied by Atul Limited, Gujarat, India. Lapox-12 is a liquid, unmodified epoxy resin of medium viscosity. Hardener k6 is a low-viscosity room-temperature curing liquid. It is generally preferred in hand-lay-up applications. Being reactive, it gives a short pot life and rapid cure at normal ambient temperatures. The reinforcement is a Roystonea regia (royal palm) fibre, which was collected from the foliage of locally available royal palm tree through the water retting and mechanical extraction.

\subsection{Fibre extraction}

From the foliage sheath, the leaves and leaf stem were removed and the sheath is dried for 3 days in shade. In the next step, it was immersed in water retting tank for 3 weeks, followed by hand rubbing and rinsing in water till the unwanted greasy material was dissolved and fine fibre was extracted. Finally, the extracted fibre once again washed thoroughly in plenty of clean water to remove the surplus waste. Continuous fibre was obtained with length up to $1.5 \mathrm{~m}$. The obtained fibre was dried under sun for 1 week. The average diameter of the fibre used for the composite preparation was between 0.2 and $0.3 \mathrm{~mm}$.

\subsection{Alkali treatment}

The dry fibre was treated with $5 \%$ solution of $\mathrm{NaOH}$ for $2 \mathrm{~h}$ to remove the unwanted soluble cellulose, hemi cellulose, pectin, lignin, etc. (Bledzi and Gassan 1999) from the fibre. The fibre to solution weight ratio was maintained at $1: 25$. After $2 \mathrm{~h}$ the fibre was washed thoroughly in distilled water to remove excess of $\mathrm{NaOH}$ and dried at $60^{\circ} \mathrm{C}$ for $24 \mathrm{~h}$.

\subsection{Chemical analysis for alpha $(\alpha)$-cellulose}

Chemical analysis was done for the determination of alpha $(\alpha)$-cellulose as per Indian standards (IS: 6213, PART III-1971) with $17.5 \%$ and $8.3 \%$ (by weight) sodium hydroxide reagents. The residual high-molecularweight fraction was left behind when a mixture of fibre and $8.3 \% \mathrm{NaOH}$ solution is filtered after the fibres have been initially swollen in a $17.5 \% \mathrm{NaOH}$ solution. The residue was washed with distilled water followed by acetic acid solution at $20^{\circ} \mathrm{C}$ and soaked for $5 \mathrm{~min}$. Again, it was washed with the distilled water to free it from the acetic acid and dried in the oven at $105^{\circ} \mathrm{C}$. Next, the 
contents were transferred to a weighing bowl and dried to a constant weight.

$\%$ alpha $(\alpha)$-cellulose can be calculated as follows:

$$
x=100 a / m \text {, }
$$

where $x=\%$ alpha $(\alpha)$-cellulose by weight; $a=$ weight of precipitate in grams; $m=$ weight of fibre in grams calculated on oven dry basis.

\subsection{Composite preparation}

The mould box was made with the dimension of $200 \mathrm{~mm}$ $(\mathrm{L}) \times 150 \mathrm{~mm}(\mathrm{~W}) \times 3.0(\mathrm{~T}) \mathrm{mm}$. The required equipment for making the mould for hand-lay-up process were glass, Teflon sticker (to cover the inner sides of the mould for easy release of the composite without sticking to the mould walls), spacer frames and a transparent plastic film to keep on the top of the uncured composite before it was closed and compressed. The treated and untreated fibres were cut according to the mould size. Then, the matrix was prepared by mixing the hardener to epoxy. The epoxy and hardener ratio was maintained at $10: 1$. To get the well-cured and a standard-quality specimen, the epoxy and hardener must be mixed smoothly and slowly for approximately $10 \mathrm{~min}$. Initial layer of the mould was filled with epoxy resin mixture and then the appropriate quantity of fibres was placed such that epoxy mixture completely spread over the fibres. Again, epoxy mixture was poured on the fibre. Thus, the starting and ending of the layers were of epoxy resin. The plastic releasing film was placed on the top of the uncured mixture. Before applying compression, efforts were made to remove all bubbles with roller. Finally, the compression pressure was applied evenly to achieve the uniform thickness of $3 \mathrm{~mm}$ and cured for $24 \mathrm{~h}$ at room temperature. The obtained composite laminates are of the size $200 \times 150 \times 3 \mathrm{~mm}^{3}$.

\subsection{Testing of the composites}

The composite specimens were tested as per ASTM standards. Tensile testing was done as per ASTM D 3039-76 with the help of INSTRON-3369 model Universal Testing Machine at a crosshead speed of $10 \mathrm{~mm} / \mathrm{min}$. The temperature was conditioned at $22^{\circ} \mathrm{C}$ with the humidity of $50 \%$. The specimen dimensions were $150 \times 15 \times$ $3 \mathrm{~mm}^{3}$. Flexural testing was done as per ASTM D 594396 standards using three point bending method at a crosshead speed of $5 \mathrm{~mm} / \mathrm{min}$ and at a temperature of $22^{\circ} \mathrm{C}$ with the humidity $50 \%$. The specimen dimensions were $100 \times 15 \times 3 \mathrm{~mm}^{3}$. INSTRON-3369 model Universal Testing Machine is specially designed for the automated material testing of polymer composites by INSTRON Corporation. The impact testing was done as per ASTM D 256-88 by Izod impact machine with unnotched specimen. The specimen dimensions were $122 \times 13 \times$
$3 \mathrm{~mm}^{3}$. In each case, five samples were tested and the average values were reported.

\section{Results and discussion}

Tensile strength, tensile modulus and \% elongation at break of untreated Roystonea regia fibre (UTRF)-reinforced and alkali-treated Roystonea regia fibre (ATRF)-reinforced composites were presented in table 1 at different fibre contents $(5,10,15$ and $20 \%$ wt.). For proper understanding of the effect of fibre content on tensile strength, tensile modulus and percentage elongation at break separate charts have been plotted for untreated and alkali-treated types of composites as shown in figures 1-3, respectively. From figures $1-3$, it is evident that with the increase in fibre content there is an increase in tensile strength, tensile modulus and percentage elongation at

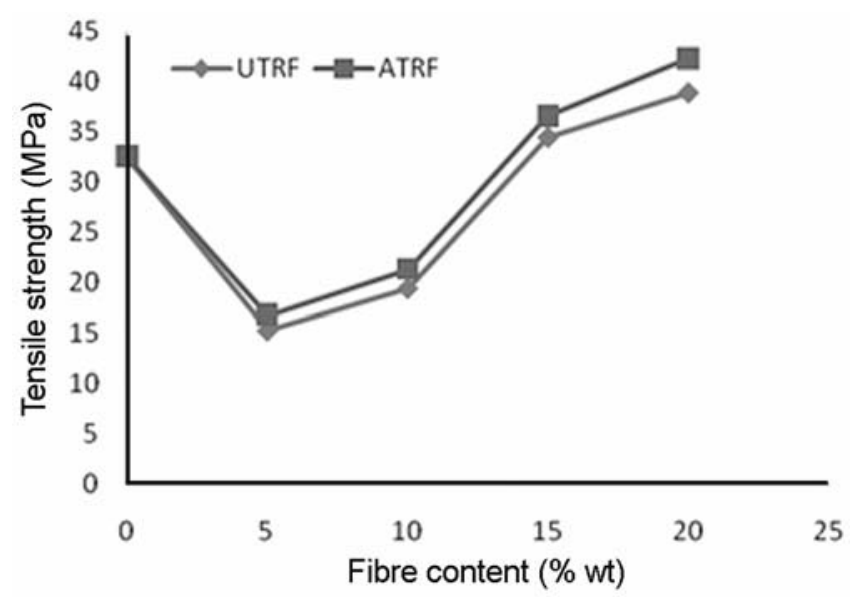

Figure 1. The effect of fibre content on tensile strength of untreated and alkali-treated composites.

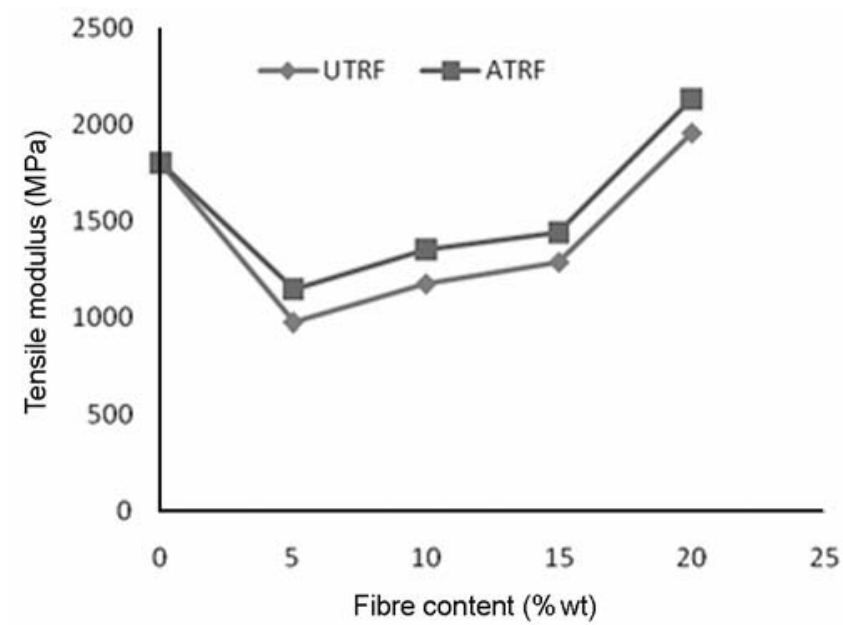

Figure 2. The effect of fibre content on tensile modulus of untreated and alkali-treated composites. 
Table 1. Tensile properties of untreated and treated composites at different fibre contents.

\begin{tabular}{|c|c|c|c|c|}
\hline Type of composite & $\begin{array}{c}\text { Fibre content } \\
\text { (\% wt.) }\end{array}$ & $\begin{array}{c}\text { Tensile strength } \\
\text { (MPa) }\end{array}$ & $\begin{array}{l}\text { Tensile modulus } \\
\quad(\mathrm{MPa})\end{array}$ & $\begin{array}{c}\text { Elongation at } \\
\text { break }(\%)\end{array}$ \\
\hline Epoxy without reinforcement & 0 & $32 \cdot 5$ & 1800 & $1 \cdot 6$ \\
\hline UTRF & $\begin{array}{l}05 \\
10 \\
15 \\
20\end{array}$ & $\begin{array}{l}15 \cdot 34 \\
19 \cdot 47 \\
34 \cdot 47 \\
38 \cdot 89\end{array}$ & $\begin{array}{r}980 \cdot 95 \\
1178 \cdot 60 \\
1289 \cdot 76 \\
1957 \cdot 31\end{array}$ & $\begin{array}{l}2 \cdot 37 \\
3 \cdot 30 \\
3 \cdot 39 \\
3 \cdot 82\end{array}$ \\
\hline ATRF & $\begin{array}{l}05 \\
10 \\
15 \\
20\end{array}$ & $\begin{array}{l}16 \cdot 78 \\
21 \cdot 29 \\
36 \cdot 56 \\
42 \cdot 29\end{array}$ & $\begin{array}{l}1149 \cdot 87 \\
1356 \cdot 72 \\
1442 \cdot 71 \\
2131 \cdot 69\end{array}$ & $\begin{array}{l}2 \cdot 49 \\
3 \cdot 51 \\
3 \cdot 71 \\
3 \cdot 92\end{array}$ \\
\hline
\end{tabular}

Table 2. Flexural properties of untreated and treated composites at various fibre contents.

\begin{tabular}{lccc}
\hline Type of composite & $\begin{array}{c}\text { Fiber content } \\
(\% \text { wt. })\end{array}$ & $\begin{array}{c}\text { Flexural strength } \\
(\mathrm{MPa})\end{array}$ & $\begin{array}{c}\text { Flexural modulus } \\
(\mathrm{MPa})\end{array}$ \\
\hline Epoxy without reinforcement & 0 & 39 & $3507 \cdot 40$ \\
UTRF & 05 & $33 \cdot 423$ & $3705 \cdot 18$ \\
& 10 & $42 \cdot 828$ & $3997 \cdot 78$ \\
& 15 & $44 \cdot 187$ & $4158 \cdot 25$ \\
ATRF & 20 & $57 \cdot 655$ & $4568 \cdot 25$ \\
& 05 & $34 \cdot 808$ & $4550 \cdot 80$ \\
& 10 & $47 \cdot 243$ & $4790 \cdot 02$ \\
& 15 & $52 \cdot 052$ & $4910 \cdot 00$ \\
& 20 & $62 \cdot 677$ & $4945 \cdot 00$ \\
\hline
\end{tabular}

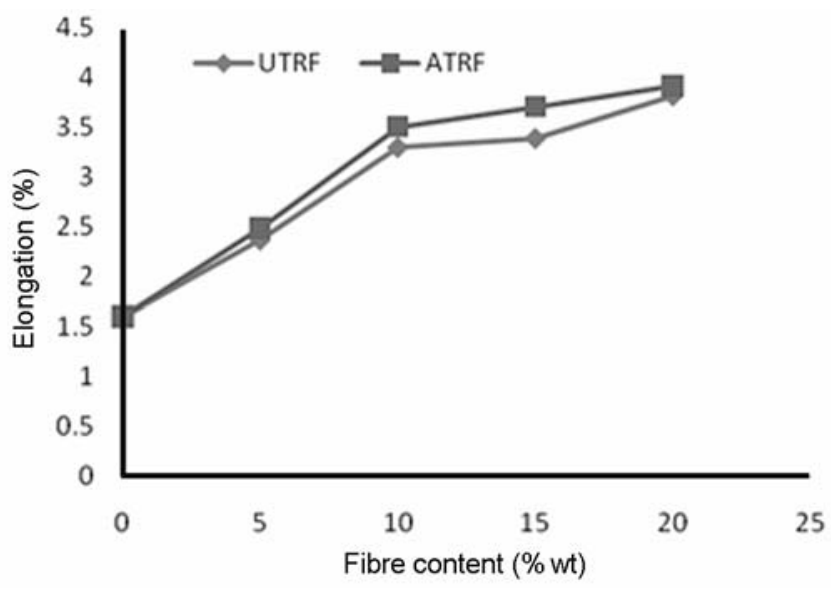

Figure 3. The effect of fibre content on $\%$ elongation at break of untreated and alkali-treated composites.

break for both treated and untreated fibre composites and the values are highest at $20 \%$ fibre content. When compared with the untreated fibre composites, the alkalitreated fibre composites recorded $8 \%$ increase in tensile strength, $8.2 \%$ increase in tensile modulus and $2.6 \%$ increase in percentage elongation at break at $20 \%$ fibre content.

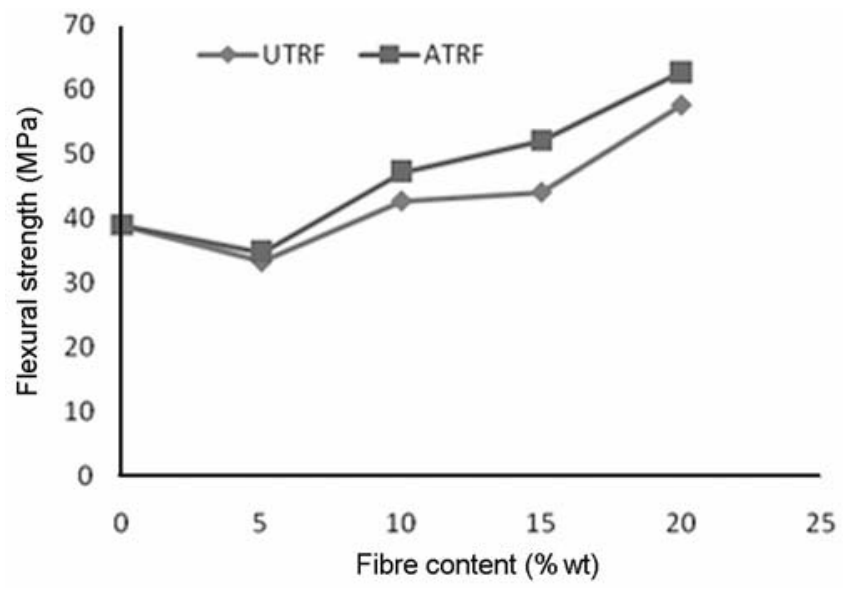

Figure 4. The effect of fibre content on flexural strength of untreated and alkali-treated composites.

Flexural properties of untreated and treated composites at various fibre contents were shown in table 2 and separate charts for flexural strength and flexural modulus are depicted in figures 4 and 5 . From the figures it can be inferred that with the increase in fibre, content, there is an increase in flexural strength and flexural modulus for both treated and untreated fibre composite which is highest at $20 \%$ fibre content. When compared with the 
Table 3. Impact strength of untreated and treated composites at various fibre contents.

\begin{tabular}{lcc}
\hline Type of composite & Fibre content $(\% \mathrm{wt})$. & Impact strength $(\mathrm{J} / \mathrm{m})$ \\
\hline Epoxy without reinforcement & 0 & 30.6 \\
UTRF & 5 & 74.36 \\
& 10 & 92.35 \\
& 15 & 116.52 \\
ATRF & 20 & 148.94 \\
& 5 & $69 \cdot 32$ \\
& 10 & 87.94 \\
& 15 & 98.06 \\
\hline
\end{tabular}

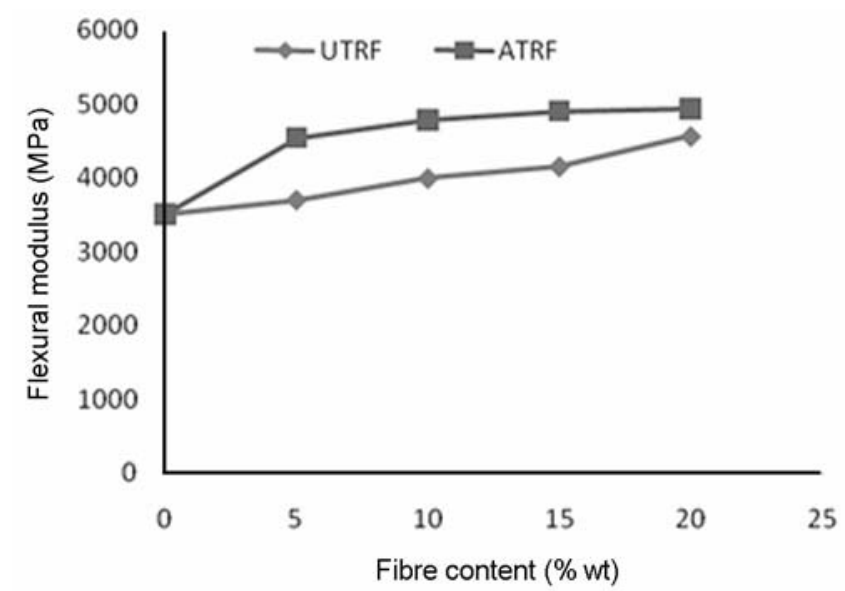

Figure 5. The effect of fibre content on flexural modulus of untreated and alkali-treated composites.

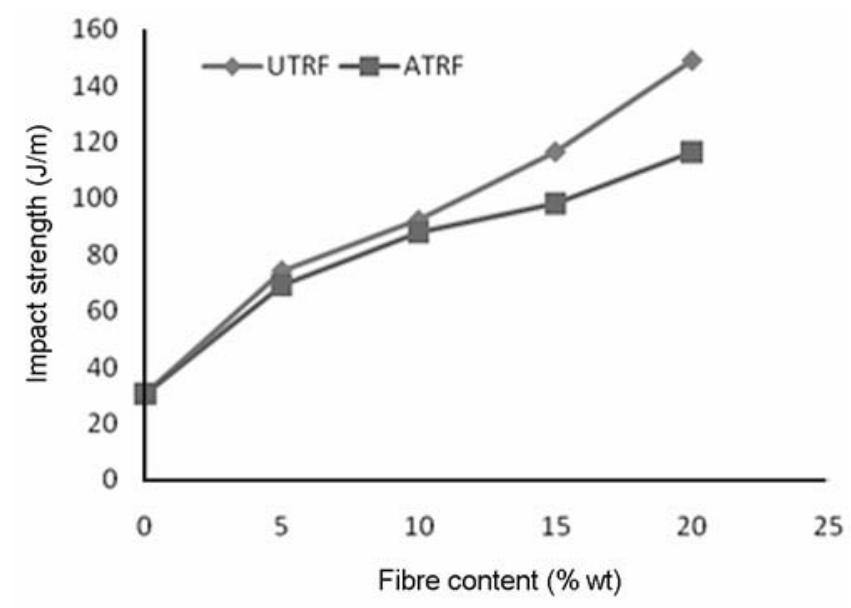

Figure 6. The effect of fibre content on impact strength of untreated and alkali-treated composites.

untreated fibre composites, the alkali-treated fibre composites recorded $8 \%$ increase in flexural strength and $7.7 \%$ increase in flexural modulus at $20 \%$ fibre content. This increase in tensile and flexural properties of alkali- treated fibre composites could be due to the increased interface between matrix and fibre after treatment. This is evidenced by the fracture morphology of tensile and flexural composites before and after alkali treatment as shown in the figures $7 \mathrm{a}-\mathrm{d}$. The fibre damage is more in untreated fibre-reinforced composites when compared with the alkali-treated fibre-reinforced composites. The alkali treatment by removing hemicellulose and lignin contents from the fibre yields the higher percentage of $\alpha$ (alpha) cellulose in natural fibres Jayaramudu et al (2009). Chemical analysis revealed that after alkali treatment, the percentage of $\alpha$-cellulose of Roystonea regia fibre increased from 58 to $65 \%$. This will render the fibre surface coarser, leading to better interface between matrix and fibre. Alkalization also causes fibrillation, i.e. breaking of fibre bundles in to smaller fibres, which would increase the effective surface area available for wetting by the matrix material (Yan et al 2000). After fibrillation due to the reduced diameter of the fibre, the aspect ratio of the fibre increases and yields rough surface topography, which in turn offers a better fibre-matrix interface. This results in obtaining the enhanced properties. The effect of alkali treatment in modifying surface of fibre can be observed by scanning electron microscopy (SEM) as shown in the figures $8 \mathrm{a}$ and b. The alkalitreated fibre has become coarser (see figure $8 \mathrm{~b}$ ) when compared with the untreated fibre (see figure $8 a$ ).

Izod impact test results were reported in table 3 for untreated and alkali-treated composites at various fibre contents and the corresponding chart was shown in the figure 6 . From the figure it is evident that although there is an increase in impact strength with increase in fibre loading, the values were decreased after alkali treatment and, at $20 \%$ fibre loading, the impact strength was decreased by $22 \%$. This could be mainly due to the reason that during the impact considerable part of energy absorption takes place through the fibre pull-out process, but after alkali treatment, due to the removal of soluble greasy contents from the fibre, strong mechanical interlocking develops between fibre and matrix, and fibre pull-out is minimized. This, in turn, will decrease the impact strength. 

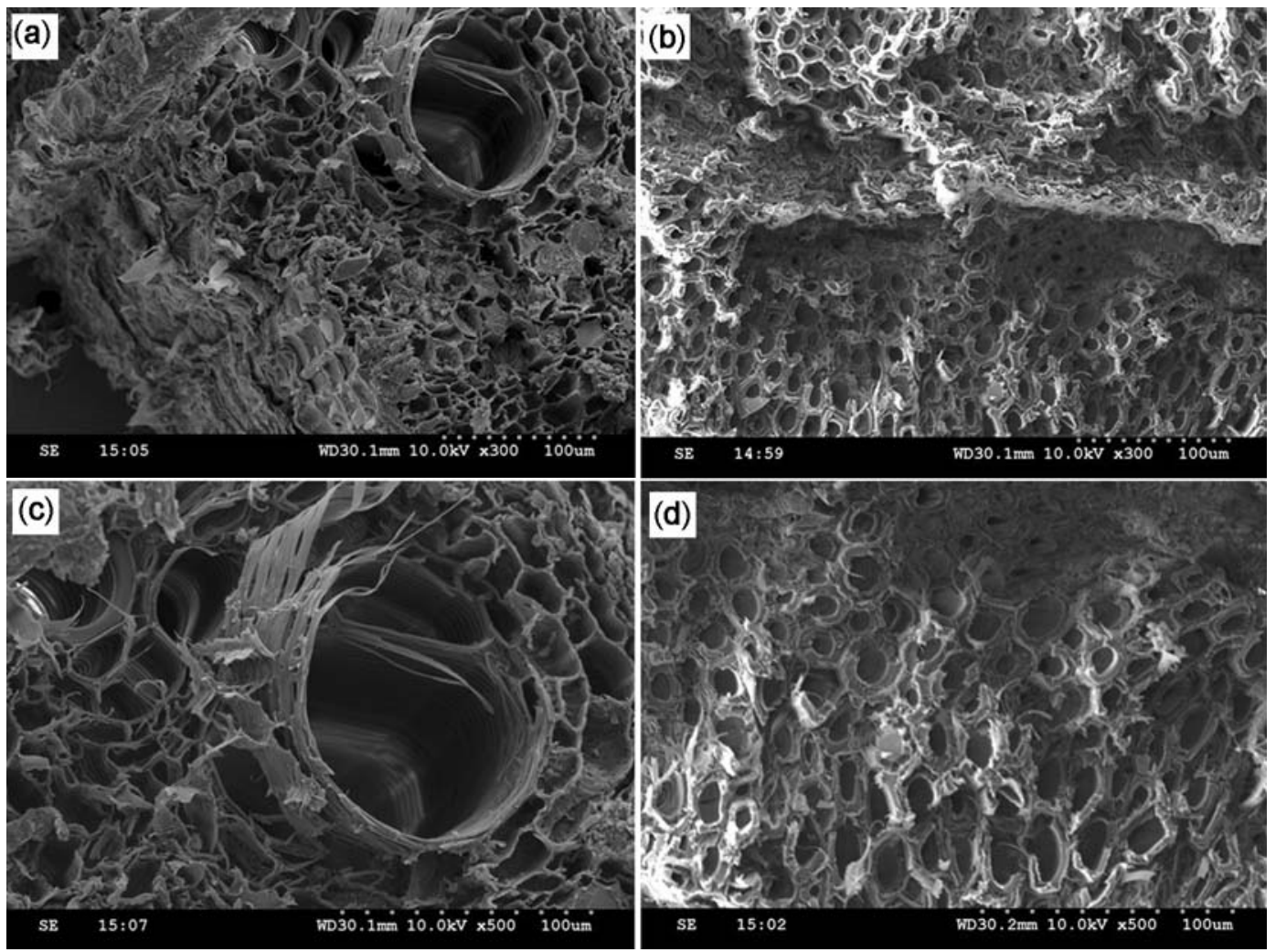

Figure 7. (a) Tensile fractured surface of untreated composite ( $20 \%$ fibre content). (b) Tensile fractured surface of alkali-treated composite (20\% fibre content). (c) Flexural fractured surface of untreated composite (20\% fibre content). (d) Flexural fractured surface of alkali-treated composite (20\% fibre content).
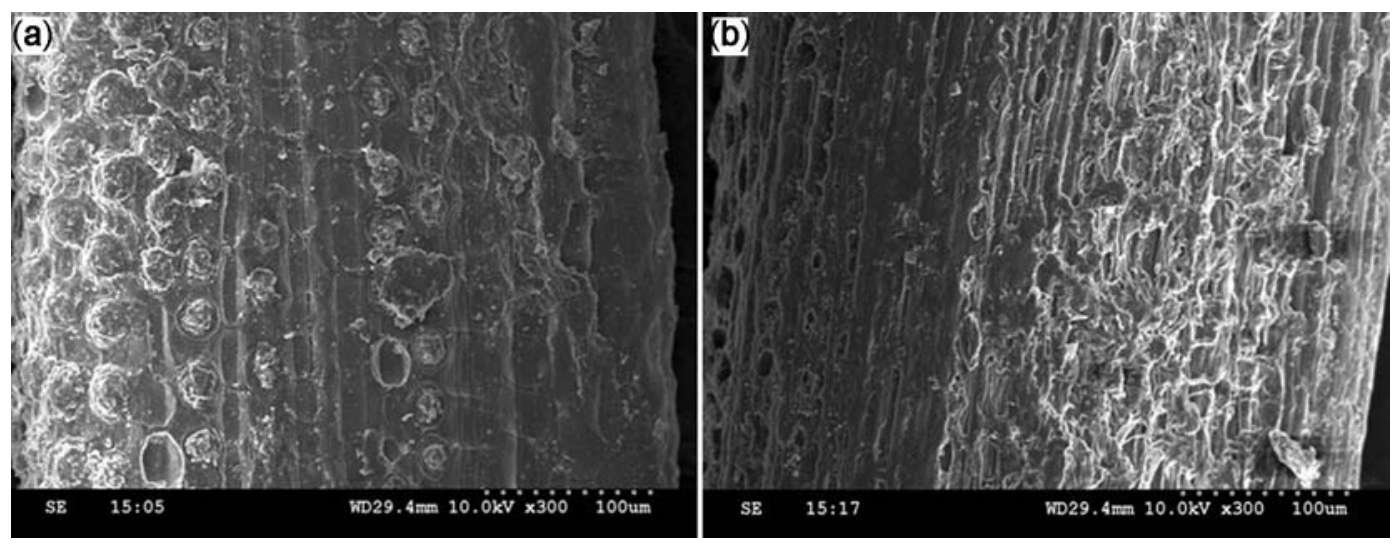

Figure 8. (a) Untreated fibre surface. (b) Alkali-treated fibre surface.

\section{Conclusions}

Tensile strength, tensile modulus and percentage of elongation of untreated and alkali-treated Roystonea regia natural fibre-reinforced epoxy composites were increased with increase in fibre content and are highest at $20 \%$ wt. fibre content. Alkali-treated fibre composites have shown superior tensile properties than untreated composites. Also flexural strength and flexural modulus of untreated and alkali-treated fibre composites were increased with increase in fibre content and are highest at $20 \%$ wt. fibre content. Alkali-treated composites have shown superior flexural properties than untreated fibre composites. For both untreated and alkali-treated fibre composites, there is an increase in impact strength with increase in fibre content and found to be highest at $20 \%$ wt. fibre content. But, the impact strength of alkali-treated fibre composites was decreased at all fibre contents when compared with the 
untreated fibre composites. Study demonstrated that Roystonea regia natural-fibre-reinforced epoxy composites could be successfully produced with good mechanical properties and the tensile and flexural properties can be further enhanced by alkali treatment. This study also reveals that maximum strength and maximum toughness can not be achieved simultaneously and optimum combinations of desired mechanical properties are possible only through careful design of composites.

\section{References}

Batchiar D, Sapuan S M and Hamdan M M 2008 J. Mater. Design 291285

Bisanda E T N and Ansell M P 1991 Compos. Sci. Technol. 41165

Bledzi A K and Gassan J 1999 Prog. Polym. Sci. 24221

George J, Bhagawan S S and Thomas S 1998 Compos. Interfaces $\mathbf{5} 201$

Guduri B R, Rajulu A V and Luyt A S 2006 J. Appl. Polym. Sci. 1021297

Huda M S, Lawrence T Drzal, Amar K Mohanty and Manjusri Misra 2008 Compos. Sci. Technol. 68424
Jayaramudu J, Guduri B R and Rajulu A V 2009 Int. J. Polym. Anal. Charact. 14115

Joseph K, Thomas S and Pavithran C 1996 Polymer 375139

Joseph S, Sreekala M S, Oommen Z, Koshy P and Thomas S 2002 Compos. Sci. Technol. 621857

Rong M Z, Ming Qiu Zhang, Yuan Liu, Gui Cheng Yang and Han Min Zeng 2001 Compos. Sci. Technol. 611437

Rao M M K and Mohana Rao K 2007 J. Comp. Struct. 77288

Rout J, Misra M, Thripathy S S, Nayak S K and Mohanty A K 2001 Polym. Compos. 22468

Rout J, Misra M, Thripathy S S, Nayak S K and Mohanty A K 2001 Compos. Sci. Technol. 611303

Shin F G, Xian X J, Zheng W P and Yipp M W 1989 J. Mater. Sci 243483

Sinha E and Rout S K 2009 Bull. Mater. Sci. 3265

Sreekala M S and Thomas S 2003 Compos. Sci. Technol. 63 861

Theepopnatkul P, kaerkitcha N and Athipongarporn N 2009 Composites B40 628

Valadez-Gonzalez A, Cervantes-Uc J M, Olayo R and HerreraFranco P J 1999 Composites B30 309

Yan Li, Yiu-Wing Mai and Lin Ye 2000 Compos. Sci. Technol. 602037 